\title{
Prospective Randomized Clinical Trial of Wide Area Circumferential Pulmonary Vein Ablation versus Segmental Pulmonary Vein Ablation for Pulmonary Vein Isolation as a Treatment for Short-Duration Paroxysmal Atrial Fibrillation
}

\author{
Sapan Bhuta ${ }^{1}$, Gustaf Sverin ${ }^{2}$, Hiro Kawata ${ }^{3}$, Malek Bashti ${ }^{1}$, Jessica Hunter ${ }^{4}$, Kathryn \\ Lewis $^{4}$, Maylene Alegre ${ }^{5}$, Amir Schricker ${ }^{6}$, David Krummen ${ }^{1}$, Gordon Ho ${ }^{1}$, Jonathan Hsu ${ }^{7}$, \\ Kurt Hoffmayer ${ }^{7}$, and Gregory Feld ${ }^{8}$ \\ ${ }^{1}$ University of California San Diego Health System \\ ${ }^{2}$ Private Practice \\ ${ }^{3}$ University of California, Irvine \\ ${ }^{4} \mathrm{UCSD}$ \\ ${ }^{5}$ University of California San Diego \\ ${ }^{6}$ Sutter Health Sacramento Sierra Region \\ ${ }^{7}$ University of California, San Diego \\ ${ }^{8}$ University of California-San Diego
}

January 29, 2021

\begin{abstract}
Background: Previous studies suggest that wide area circumferential pulmonary vein ablation (WACA) is more effective than segmental pulmonary vein ablation (SPVA) for pulmonary vein isolation (PVI) for treatment of atrial fibrillation. Whether this is true in patients (pts) with very short duration paroxysmal atrial fibrillation (PAF) is unknown. Objective: To compare WACA to SPVA in pts with PAF lasting $<48$ hours. Methods: One hundred pts with PAF $<48$ hours were randomized to either WACA vs SPVA (45 and 53 pts respectively, with 2 withdrawals), and followed up for 24 months with 14-day ECGs every 6 months. Results: Among $97 \mathrm{pts}$ at an average of 22.1 4.8 months followup, 26 (57.8\%) remained free of any atrial arrhythmias after WACA versus $29(55.86 \%)$ after SPVA $(\mathrm{p}=0.64)$. Sixteen pts $(35.6 \%)$ had recurrent PAF after WACA versus 20 pts $(38.5 \%)$ after SPVA $(\mathrm{p}=0.79)$. Seven pts $(15.6 \%)$ had atrial flutter after WACA versus 5 pts $(9.64 \%)$ after SPVA ( $=0.376)$ and $1 \mathrm{pt}(2.2 \%)$ had atrial tachycardia after WACA vs $1 \mathrm{pt}(1.9 \%)$ after SPVA $(\mathrm{p}=0.918)$. Total procedure time was lower for SPVA vs WACA (242.9 vs 271.1 minutes, $\mathrm{p}=0.047$ ), and fluoroscopy time similar for WACA vs SPVA (50.8 vs 53.4 minutes, $\mathrm{p}=0.555)$. Conclusions: As an initial ablation approach in pts with PAF $<48$ hours, SPVA was similarly effective to WACA with respect to arrhythmia recurrence, supporting the central role of the pulmonary veins for maintaining AF in these pts. Future therapies using alternative ablation energies may incorporate these insights to reduce risk to gastroesophageal structures.
\end{abstract}

\section{Hosted file}

WACA vs SPVA for PAF Final Draft.pdf available at https://authorea.com/users/392662/articles/ 506434-prospective-randomized-clinical-trial-of-wide-area-circumferential-pulmonaryvein-ablation-versus-segmental-pulmonary-vein-ablation-for-pulmonary-vein-isolation-asa-treatment-for-short-duration-paroxysmal-atrial-fibrillation 

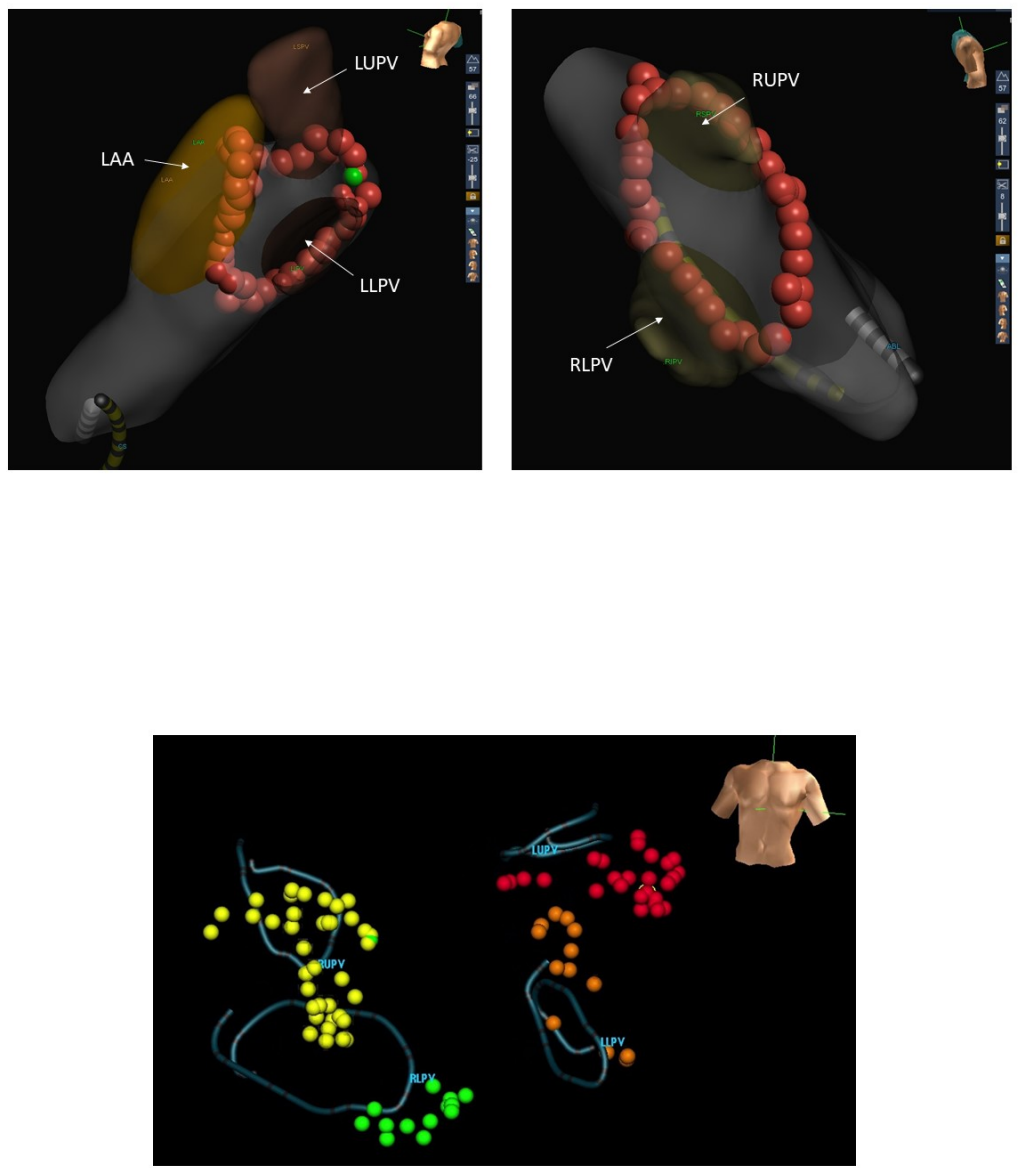


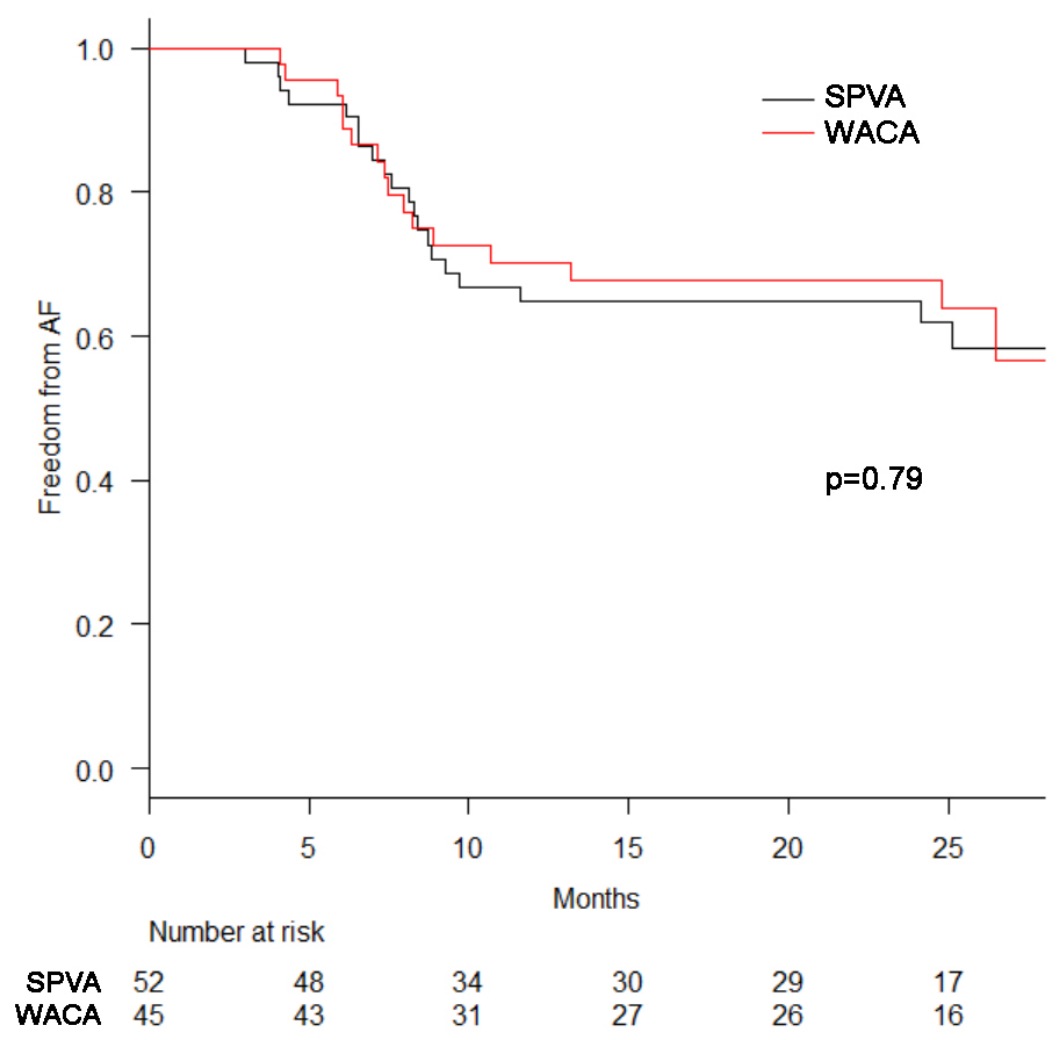




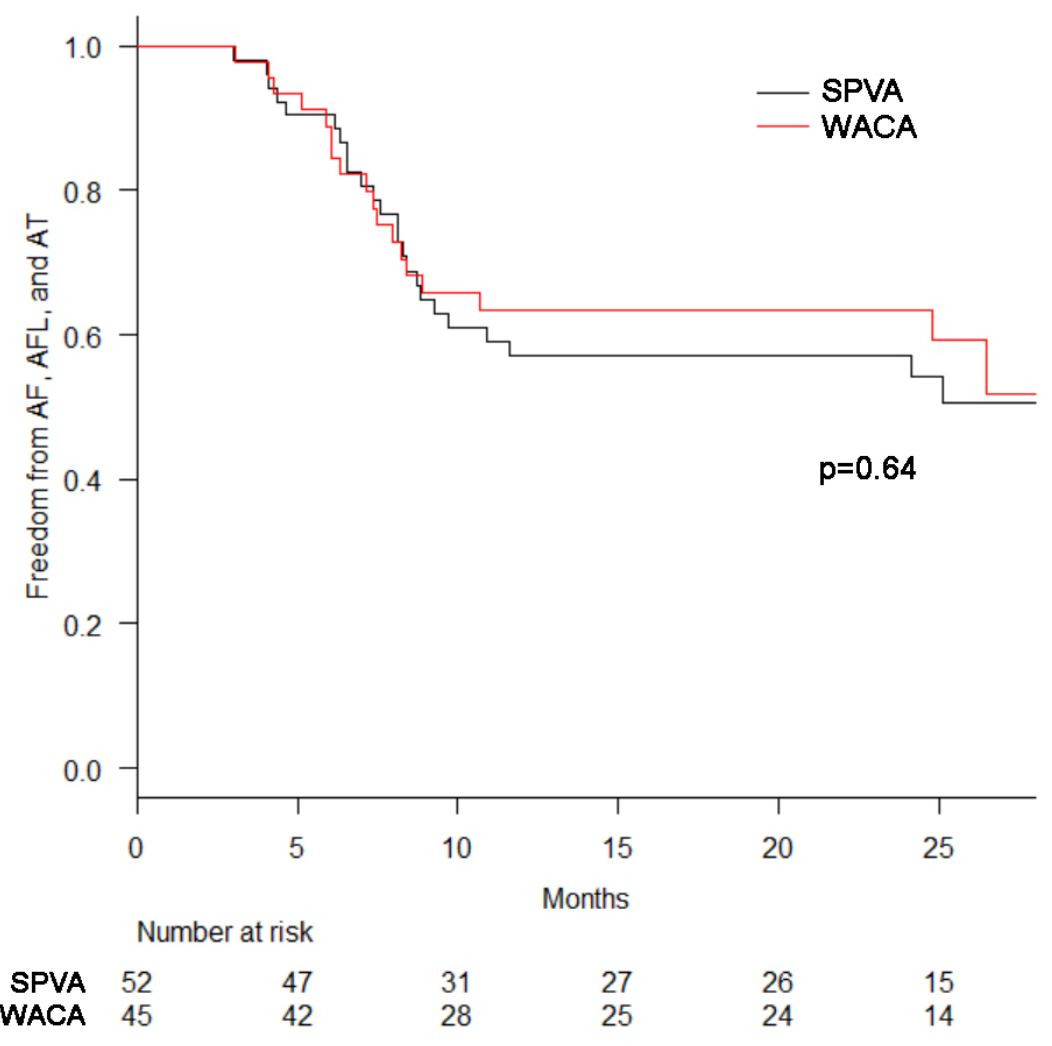

